\title{
DISCOURS ENVIRONNEMENTAL ET MISE EN SCÈNE DE LA SOLIDARITÉ
}

\author{
Le cas du Grand Lyon
}

Valérie Colomb, Dana Popescu-Jourdy, Elizabeth Vercher ${ }^{1}$

Ce travail se trouve à l'articulation entre une manière de mettre en politique le développement durable et les manières de le dire : entre un discours institutionnel et des pratiques solidaires qui se font au nom du développement durable. Notre approche part de l'étude des espaces verts dans le projet politique du Grand Lyon qui propose une redéfinition de son identité à travers la problématique environnementale.

Les problématiques environnementales sont au cœur des préoccupations des collectivités territoriales. La communauté urbaine du Grand Lyon s'inscrit dans ce mouvement. Depuis 2001, elle affiche la question du développement durable comme un axe politique structurant de son projet territorial. Les édiles et les techniciens de la collectivité s'approprient progressivement les nouveaux enjeux et réactualisent, au nom

1 Valérie Colomb est maître de conférences à l'IEP de Lyon ; Dana Popescu-Jourdy est maître de conférences à l'ICOM, université Lumière Lyon 2 ; Elizabeth Vercher est maître de conférences à l'ICOM, université Lumière Lyon 2.

Recherches en communication, $\mathrm{n}^{\circ} 35$ (2011). 
du développement durable, des actions préexistantes dans le domaine de l'environnement. D'un point de vue opérationnel, ils inventent des dispositifs concrets sous couvert de l'approche heuristique postulée par ce concept. Dans un même temps, l'institutionnalisation progressive du développement durable mobilise de plus en plus des discours sur la solidarité en lien avec le fait de favoriser les aménités urbaines dans toute leur complexité.

Les créations de la Charte de l'arbre en ville (2000, remaniée en $2011)^{1}$ et de l'Observatoire du développement durable $(2005)^{2}$ ainsi que les expériences autour des Murs végétalisés et le projet Le jardin citoyen, sont autant d'initiatives qui allient à la fois une approche environnementale et la volonté de trouver des traductions concrètes à la notion de solidarité. Les discours d'accompagnement de ces projets s'insèrent à leur tour dans ce que nous pouvons appeler la communication environnementale du Grand Lyon, centrée sur un axe fédérateur des valeurs éthiques fortes. Ainsi, les acteurs construisent discursivement une dimension environnementale de leur territoire autour de la notion de la solidarité.

Notre recherche vise à analyser comment la communication environnementale relève de plus en plus d'une démarche d'engagement et de pédagogie. Une fois le principe de la responsabilité solidaire reconnu par les acteurs du territoire, le projet environnemental constitue une médiation autour de nouvelles pratiques et de discours émergents.

Ce travail se trouve à l'articulation entre les manières de mettre en politique le développement durable, notamment la question des espaces verts, et les manières de dire les projets, à savoir entre des pratiques solidaires qui sont proposées au nom de l'environnement et des discours institutionnels qui tentent de redéfinir l'identité territoriale et le lien territorial à travers la problématique environnementale.

1 http://www.grandlyon.com/fileadmin/user_upload/Pdf/activites/environnement/ Arbres/Charte_arbre.pdf (dernière consultation le 19 décembre 2010).

2 Dans le cadre de cet observatoire, des cahiers thématiques sur le développement durable ont été élaborés comme outil de réflexion et de travail : en 2009, un cahier sur le volet économique, en début 2011 un sur le volet environnemental et en fin 2011 sur le volet social. 


\section{Les enjeux d'une articulation entre environnement et solidarité}

Plusieurs grandes rencontres internationales, textes fondateurs, normes et labels, récits pédagogiques, slogans, schémas et figures emblématiques ont assuré une forte focalisation médiatique et la prospérité de l'expression " développement durable », qui trouve ses fondements dans les années 70 à 90 . La construction discursive par les reprises et la transformation des énoncés qu'elles permettent sont en lien avec les pratiques qui se mettent en place au nom du développement durable. L'espression fonctionne d'un point de vue linguistique comme une formule (Krieg-Planque, 2009), un méta-récit (JalenquesVigouroux, 2006), un oxymore, une conception éco-systémique de la communication (Libaert, 2010) mais aussi comme un mythe rationalisateur et un référentiel (Porcedda, 2009), une idéologie, une utopie ou un modèle de société ayant pour but une qualité de vie pour tous (Tremblay, 2007).

Toutefois, en matière d'urbanisme, la définition de G.H. Brundtland fournie en $1987^{1}$ avec ses trois « piliers »- économie, société, environnement - sert le plus souvent de matrice à la réflexion opérationnelle.

En tant que coproduction de la société civile, de l'Etat, des collectivités territoriales et du monde de l'entreprise, à l'échelle internationale, le développement durable s'exprime par des pratiques et des enjeux très variés selon les acteurs, les dispositifs normatifs et les lois applicables d'un secteur concerné. Le développement durable dans l'entreprise avec, par exemple, le reporting mis en place au nom de la Responsabilité Sociale des Entreprises, ne se manifeste pas selon les mêmes logiques et temporalités que dans une commune avec les outils de l'Agenda $21^{2}$. C'est en tant que guidage pour des démarches opérationnelles renouvelées et de transformation de la ville qu'il nous intéresse ici. En effet, une fois la demande sociale élaborée et la valeur désirable constituée

1 En 1982, l'ONU commande un rapport sur l'écodéveloppement à Mme Gro Harlem Brundtland qui sera remis en 1987, avec le titre de Rapport des Nations Unies pour la Commission Mondiale sur l'Environnement et le Développement (CMED).

2 L'Agenda 21 local porté par une collectivité est un plan d'actions global pour mettre en œuvre le développement durable à l'échelle d'un territoire. Son élaboration se conduit dans le cadre d'une concertation entre des parties prenantes élargies. 
chez les élus, il s'agit pour la collectivité de construire politiquement le développement durable et de mettre en acte la formule. D'autre part, les manifestations communicationnelles du développement durable pour la ville et sa temporalité longue ne recouvrent pas le même degré de maturité que pour la publicité.

Nous pensons que la mise en politique du développement durable reparamètre et réactualise les pratiques et les discours de solidarité qu'il convient de saisir, notamment en tenant compte du fait que la notion de solidarité se constitue à son tour dans une sorte de grand récit et participe, à travers les discours des acteurs qui l'utilisent, à une mise en scène particulière de leur identité fondée sur des formes spécifiques de participation, d'engagement et de médiation autour des projets.

Par son étymologie, le mot «solidarité » renvoie à un principe juridique. Le latin solidus se rapporte à l'interdépendance des débiteurs entre eux. Chacun est engagé, en termes de dette et de responsabilité, pour le tout (in solidum). Le concept - qui rejoint sans doute l'idée pythagoricienne du nombre - insiste sur une totalité, une unité dont la sollicitude s'exerce sur chaque être qui la constitue.

La solidarité confère aux projets et aux acteurs participants la preuve de leur légitimité et favorise leur visibilité dans l'espace public. Les participants à un projet à vocation explicitement environnementale mettent en scène une forme de rationalisation - dans le sens wébérien du terme - de l'action solidaire et cette rationalité constitue un cadre qui va définir à son tour des discours et des pratiques spécifiques de communication : dans notre cas, la communication environnementale de la ville.

\section{Ville, nature et pratiques de solidarité}

Historiquement, la présence de la végétation en ville relève essentiellement des jardins privés : un agrément au service des nobles et notables. Il faudra attendre la Révolution française pour voir apparaître le jardin public en France. Le jardin privé devient un bien national. L'accès à ces espaces verts induit de nouveaux usages urbains. Les différences sociales s'effacent face à une solidarité implicite par un accès à ces espaces ouverts à tous. Au XIXème siècle, une pensée renouvelée de la ville voit la plantation d'arbres d'alignements en quantité le long des tous nouveaux boulevards, et la création de parcs, places et squares. 
Avec la ville haussmanienne, le végétal tente de répondre à des enjeux sanitaires et d'embellissement qui vont s'inscrire rapidement dans les politiques publiques. Il faut retrouver de l'ombre et une certaine qualité de vie. La nature est ici prophylactique et on est proche de la logique du solidarisme. Parallèlement, on assiste à l'émergence du tourisme et à un engouement pour des évènements ludiques autour des fleurs - nouveau lieu du montrer social - pour distraire une riche clientèle oisive. La dimension sociale de ce mouvement suivra avec des incitations pour le fleurissement des espaces publics et des logements sociaux par des actes de philanthropies (dons de plantes aux plus pauvres). Des pratiques émergentes dans les classes privilégiées se diffusent dans le corps social. Tout le monde se doit de participer, de manière solidaire, à cet effort.

D'autre part, sur le modèle du clos du pauvre de Saint Vincent de Paul au XVIIème siècle, le jardin « solidaire » se repense au XIXème sous l'intitulé de jardin ouvrier. D'approche religieuse et morale, paternaliste et sociale, il entretient un lien à la terre, procure un complément alimentaire à des populations rurales venues travailler en ville et éloigne les ouvriers des ravages de l'alcool. Durant le XXème siècle, on passe de la prise en compte d'une solidarité par la philanthropie en lien avec le religieux à une solidarité organisée par les politiques publiques : une forme de laïcisation de la solidarité.

Selon E. Boutefeu (2008), au début du XXème siècle, la question de l'organisation de la ville pour assurer à tous les citadins un accès à la nature se pose à travers deux modèles :

- les cités jardins horizontales, ou placer la nature au cœur des villes avec un risque d'étalement urbain

- les cités jardins verticales, ou construire en hauteur avec des toits terrasses pour éviter ce risque.

Evidemment, ces deux approches n'épuisent pas à elles seules les modèles sur la place de la nature en ville. En effet, dans les années 1950, l'émergence des loisirs stimule un urbanisme vert polarisé sur la création de grands parcs en périphéries des villes : on parle de ceinture verte. La collectivité, par ce modèle d'espace ouvert, offre à tous les habitants un contact avec la nature.

La réflexion sur la nature en ville prend du retard au XXème siècle. La ville se construit contre la nature et contre ses habitants, et cela jusqu'aux années 1980 : «la ville devient minérale, polluée, bruyante, accidentogène (...) une ville centrifuge avec la désertification des 
centres et une recherche en périphérie d'un contact avec la nature $»^{1}$. Les lois de décentralisation des années 80 renforcent les ségrégations sociales par les égoïsmes municipaux et la compétition entre territoires qu'elle génère ${ }^{2}$. Parallèlement, de multiples initiatives et débats - «plan vert », "plan de végétalisation », « campagne urbaine ", « ville écologique », « ville durable »- marquent l'émergence de nouveaux registres discursifs et argumentaires sur le lien entre nature en ville et la solidarité. Ils sont autant de jalons dans cette quête de la ville durable et des nouvelles expressions de solidarité. Mais il faut attendre les années 90 , pour que le jardin, entre temps devenu un lieu de loisir, redevienne un lieu où la solidarité y est pensée. La loi de 1998 relative à la lutte contre l'exclusion définit le jardin d'insertion. Nommé « Jardin de la solidarité » ou « Jardin cultivons la solidarité », il a pour objectif d'inscrire la personne en difficulté dans un réseau de solidarité selon une insertion sociale (axe privilégié : fonction thérapeutique) ou une insertion économique (axe : réintégration dans le monde économique).

D'autre part, une proposition de loi en 2003, tente de définir des catégories : le jardin familial autrefois appelé jardin ouvrier, le jardin d'insertion et ou, le plus récent, le jardin partagé. Même si cette loi ne voit pas le jour, l'initiative marque l'intérêt du législateur pour l'expression de la solidarité dans le cadre environnemental. La politique de la ville va intégrer le jardin partagé qui crée du lien social par le biais d'activités culturelles (lieu festif et artistique), sociales (rencontres intergénérationnelle et multiethnique), éducatives (transmission de connaissances, de savoir-être et de valeurs) autour de la nature. Le lien entre nature et pratiques de solidarité va s'institutionnaliser avec le Grenelle de l'environnement et son plan Ville durable. En 2010, le plan « Restaurer et valoriser la nature en ville $»^{3}$ a pour objectif le développement des jardins solidaires. La convergence entre les piliers de l'environnement et la solidarité s'inscrit désormais de manière constante dans les discours publics.

1 Entretien avec Fréderic Ségur responsable du service Direction de la voirie Ingénierie, Arbres et Paysage, 11 octobre 2011.

2 Desjardins, X. (dir. P. Boino), L'intercommunalité et décentralisation, Paris, Plan Urbanisme Construction Architecture, juin 2006.

3 http://www.developpement-durable.gouv.fr/Plan-nature-en-ville.html (dernière consultation le 28 décembre 2010). 


\section{Le Grand Lyon et sa communication de solidarité environnementale}

Face à l'injonction politique de concrétisation du développement durable, les techniciens de la collectivité doivent inventer une démarche opérationnelle et réorganiser l'ensemble des services de façon transversale pour décloisonner l'approche sectorielle. La montée en compétence de l'ensemble des personnels devient un enjeu majeur. La démarche d'adhésion qui est avant tout une démarche culturelle et communicationnelle fait partie intégrante du processus. D'autre part, le pilier " participation et gouvernance » a été ajouté par les édiles grand-lyonnais aux entrées canoniques proposées par les documents de l'ONU dans les années 80 : social, environnemental et économique. La vision déhiérarchisée entre les piliers du développement durable a du mal à trouver son expression opérationnelle tant l'approche holistique a du mal à s'élaborer. Les acteurs s'en saisissent graduellement : le pilier « social » ne trouve ses traductions que très récemment. La solidarité semble en être une porte d'entrée privilégiée.

L'identification d'un thème global « nature en ville » commence dans les années 90. Plusieurs outils sont autant de points d'appui pour la convergence de la question environnementale et de la solidarité : première Charte de l'écologie (1992), l'Agenda 21 qui propose la création de la Charte de l'arbre en ville (1995), seconde Charte de l'écologie avec l'intégration d'un volet éducatif (1997), la deuxième édition de la Charte de l'arbre en ville (2000). En 2011, d'autres notions guident la Charte de l'arbre: la solidarité, l'éducation et l'innovation. La solidarité regroupe ici quatre axes de travail :

- la solidarité territoriale avec l'accessibilité pour tous à la nature et donc un maillage fin du territoire avec des espaces verts

- la solidarité trans-générationnelle : " on plante pour les générations futures "

- la solidarité d'échanges de savoirs entre agglomérations locales et internationales

- la solidarité symbolique avec l'utilisation de la valeur symbolique de l'arbre pour développer la solidarité notamment via le mécénat.

L'opération des «Arbres de la solidarité », organisée en partenariat avec l'association Foyer de Notre Dame des Sans-abris et plusieurs entreprises privées, a été intégrée à ce dernier point. Le Grand Lyon 
plante à ses frais un arbre. Une entreprise s'engage dans le cadre du mécénat à donner mille euros à l'association en contrepartie de son logo sur un panneau d'affichage près de l'arbre. Sa plantation peut donner lieu à un évènement de communication interne dans l'entreprise et participe aux dispositifs RSE. L'argumentaire « un geste solidaire et un geste écologique » renvoie aux deux piliers - environnemental et social - du développement durable.

A partir de la définition de la solidarité évoquée précédemment et de la mise au point sur l'évolution des projets concernant les espaces verts dans la ville, nous avons choisi de nous intéresser au cas du Grand Lyon, notamment à travers la question des jardins ouvriers. Nous nous pencherons sur la mise en scène de ces projets au travers des différents supports de communication mais aussi dans les discours qu'ils développent. Pour une question de temps et de cohérence nous avons choisi d'étudier les sites internet du Grand Lyon, Millénaire 3 (centre de ressources prospectives du Grand Lyon) et les travaux publiés en ligne du groupe Prospective $2020^{1}$ qui sont les principaux vecteurs de communication de la politique globale actuelle du Grand Lyon. Nous avons procédé à une analyse des discours des sites concernant les thématiques évoquées.

Dans ce corpus, nous nous sommes aperçues que la solidarité tient une place essentielle et qu'elle se décline souvent au travers d'actions intégrées dans le développement durable. La prospective 2020 a pour ambition première de répondre à la question suivante : « Comment faire société ? $»^{2}$. Le premier constat est que la condition principale pour construire une société humaine est l'existence d'une solidarité entre ses membres. Celle-ci se décline à travers un contrat social qui privilégie l'interdépendance intergénérationnelle et le dialogue au travers du sentiment d'appartenance à un territoire commun, indifféremment du milieu social. La solidarité n'est pas une notion acquise, ni une évidence, elle est en perpétuelle construction et faite de disparités liées à notre société avec lesquelles il faut conjuguer. Vouloir construire une aggloméra-

1 http://www.grandlyon.com/, http://www.millenaire3.com/, Synthèse des différents groupes de travail prospective Lyon 2020, document pdf en ligne sur le site millénaire 3.

2 Synthèse du groupe de travail « Solidarité et dialogue », Lyon 2020, mars 2007. http://www.grandlyon.com/.../Lyon_2020/Groupes_travail/GT_solidarite.pdf (dernière consultation le 19 décembre 2010 ). 
tion solidaire, c'est trouver des actions quotidiennes qui permettent de rassembler la différence autour de projets communs ${ }^{1}$.

La première indication de l'importance accordée à la solidarité est la place qu'elle occupe, notamment par le fait qu'elle fait partie des 9 emblèmes du Grand Lyon ${ }^{2}$. Sous le terme "solidarité » on retrouve les préoccupations liées au développement de la pauvreté et aux questions d'insertion sociale. La représentation de la solidarité se fait au travers d'un imaginaire relié à une photo, celle d'une ronde de mains. Comme on le verra, souvent dans les images présentes qui servent d'illustration au discours, on fonctionne plus sur des dispositifs basés sur l'imaginaire. Par exemple, quand on parlera de jardins, on verra un arbre ou une cabane de jardin, pour le lien social on verra un groupe de personnes.... Au récepteur d'imaginer ce qu'il voit dans ces photos. De toute façon, il est difficile de mettre en scène une réalité de ces termes, on fonctionne donc par association d'un ensemble d'éléments (Barthes, 1965). Par exemple, je parle de développement durable, je dessine un gros arbre, de la pauvreté, un bidonville ou des papiers gras par terre (connotations des « piliers » du développement durable).

On s'aperçoit aussi que nous sommes dans un dispositif de communication qui fonctionne sur deux niveaux : le premier niveau est fédérateur et vise à mobiliser la population locale autour d'un projet ; le deuxième niveau est centré sur l'institution elle-même et son principal but est d'améliorer l'image du Grand Lyon à travers l'outil de communication. Il faut aussi dire que cette démarche du Grand Lyon et son engagement dans le développement durable au travers de la solidarité s'inscrit pleinement dans la stratégie promue par le ministère. Le forum « Le développement durable, créateur de lien social » organisé à Bordeaux en août $2010^{3}$ illustre notre problématique sur le cas du Grand Lyon. Le thème qui nous intéresse, celui des jardins collectifs, trouve sa place dans les thèmes évoqués lors de cet événement par les associations et les collectivités dans leur engagement autour de 3 thèmes :

- la nature en ville, créatrice de lien social

- l'économie sociale et solidaire, un système d'économie locale et

1 www.millenaire3.com/uploads/tx_ressm3/Lyon2020_solidarite.pdf (dernière consultation le 19 décembre 2010).

2 Fleuve, gastronomie, lumière, mode, santé, solidarité, cinéma et nouvelles images, danse et arts vivants, sport. http://www.millenaire3.com/.

$3 \mathrm{http}: / / \mathrm{www}$.developpement-durable.gouv.fr/Forum-de-Bordeaux-ledeveloppement,18181.html (dernière consultation le 23 décembre 2010). 
- habiter autrement. 1

Il s'agit aussi de mettre en image la question de la solidarité à travers l'environnement, car il devient ici un moyen de réactualiser la notion d'action solidaire.

Les actions engagées s'intègrent à la fois dans une stratégie de développement durable global (promue à l'echelle nationale), dans un environnement local (celui de ville), ici à l'échelle du Grand Lyon et enfin elles nécessitent le développement d'une pédagogie de projet « sérieuse » comme nous allons le voir maintenant avec l'exemple des jardins collectifs de la région lyonnaise.

L'Agenda 21 du Grand Lyon voté en $2005^{2}$ donne une place importante aux jardins collectifs. Ils permettent l'amélioration du cadre de vie et deviennent des lieux de pratiques pédagogiques vis-à-vis de l'environnement. Ce sont des lieux d'échanges, de cohésion sociale, où différentes populations (âge, mixité sociale) cohabitent.

Dans un premier temps le projet de création d'un jardin se fait autour d'une méthodologie rigoureuse qui commence par la définition de ce que l'on souhaite faire en fonction du public visé. Les questions pratiques telles l'aménagement, le lieu, l'investissement initial sont primordiales. Ces jardins sont définis par une proposition de loi adoptée par le Sénat en 2003 et se répartissent en 3 catégories : les jardins familiaux ou jardins ouvriers, les jardins d'insertion, les jardins partagés ${ }^{3}$.

Sur le site du Grand Lyon, on voit un véritable effort de communication dans le discours pour impliquer la population. Le titre de la page,

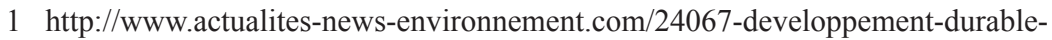
cree-lien-social.html (dernière consultation le 23 décembre 2010).

2 « Document stratégique et opérationnel pour le long terme qui vise à mettre en œuvre sur un territoire donné les principes du développement durable. A partir d'une réflexion collective sur le devenir de ce territoire, il fixe un projet (objectifs) décliné en programmes d'actions (moyens) et il est soumis à une évaluation régulière. Ce projet global est le fruit d'une large participation citoyenne. Son élaboration implique nécessairement une réflexion transversale entre les différents services et les différentes politiques, la mise en place d'un réseau d'acteurs, d'expertise et de savoir. " http://www.grandlyon.com/L-Agenda-21-du-GrandLyon.34.0.html http://www.grandlyon.com/L-Agenda-21-du-Grand-Lyon.34.0.html (dernière consultation le 22 décembre 2010).

3 «De l'intérêt des jardins et du jardinage pour l'habitat social et la santé psychique », Béatrice Charre (le passe-jardins) et Mireille Lemahieu (CAUE du Rhône), Millénaire 3. 
«Un jardin dont vous êtes le héros $»^{1}$, pourrait presque se comprendre comme le « Parce que vous le valez bien » de L'Oréal. Le héros, c'est « vous » par votre implication et votre engagement. L'utilisation de la deuxième personne dans le message vise à impliquer plus fortement le destinataire (Maingueneau, 1998) ${ }^{2}$. L'inclusion de la cible au cœur du message (Libaert, 2008) ${ }^{3}$, ainsi que le jeu entre le « vous » et le « nous », participent à la récréation d'un « collectif» et d'une « solidarité ».

L'enjeu pédagogique de ces jardins est souligné par cette phrase (Millénaire 3) :

« Le jardin devient un espace de participation démocratique et permet la création de lien social intergénérationnel et interculturel. De plus, ces jardins collectifs deviennent des lieux pratiques d'éducation à l'environnement où l'on peut aborder concrètement la gestion des déchets, la gestion économe de l'eau, l'appropriation des lieux et l'embellissement des espaces extérieurs ».

A partir de cet exemple nous pouvons voir comment le Grand Lyon met en scène sa communication. Les images et le discours laissent une grande place à l'imagination du destinataire pour construire sa propre réalité de ce qu'est la solidarité et sur le rôle qu'il a à jouer dans le projet de l'institution. L'institution, elle, améliore son image « corporate » en s'intéressant au concept du développement durable tout en introduisant la notion de « proximité participative », qui est valorisante pour la communauté qui s'implique dans le projet environnemental.

Ainsi l'environnement, relayé par communication de l'institution, devient un outil fédérateur autour de valeurs fortes telle la solidarité, le lien social ou le comportement éthique et responsable. Il existe un lien entre les pratiques communicationnelles et les pratiques sociales, les premières permettant aux secondes d'être mieux intégrées et perçues, dans les politiques locales et environnementales. La solidarité relève à la fois d'une approche économique et d'une approche morale et éthique,

1 http://www.grandlyon.com/Info.1164+M5a5eae47eaf.0.html (dernière consultation le 28 décembre 2010).

2 Maingueneau D., Analyser les textes de communication, Editions Dunod, Paris, 1998.

3 Libaert T., Communiquer dans un monde incertain, Paris, Ed Pearson, 2008. 
dans des logiques de grands récits qui se croisent au gré des discours et des pratiques.

\section{CONCLUSION}

La révolution pacifique du développement durable «préconise la construction de nouvelles solidarités capables de repousser les risques de désintégration des sociétés en une nébuleuse d'égocentrismes et de fanatismes de tous genres $»^{1}$ et l'écogestion urbaine est en soi considérée par les élus comme un processus éducatif. Le discours environnemental appliqué à la ville est un discours à forte dimension pédagogique. L'insertion du paradigme de la communication solidaire dans la communication environnementale signe la consolidation discursive dans les pratiques du volet social du développement durable. Les changements et les outils de réflexion sur notre société urbaine sont bouleversés tout comme la communication qui l'accompagne. Cependant, la représentation iconique de la solidarité souffre pour l'instant d'un manque de visibilité. La solidarité environnementale se dit, se pratique et commence à se voir.

1 Lefèvre P., Voyages dans l'Europe des villes durables, Paris, PUCA CERTU, 20. 


\section{Références}

Barthes, R., Eléments de sémiologie, Denoë1/Gonthier, Paris, 1965.

Bernoux, P. (2004). Sociologie du changement, Paris : Ed. du Seuil.

Bertin, E. (2007). Penser la stratégie dans le champ de la communication. Une approche sémiotique. Nouveaux actes sémiotiques, 110 (on line).

Blais, M.-C. (2007). La solidarité. Histoire d'une idée. Paris : Gallimard.

Boutefeu, E. (2008). Brève histoire contemporaine de l'urbanisme végétal. mis on-line le 09-06-2008, site du Centre d'Etudes des Réseaux de Transports et d'Urbanisme http://www.certu.fr/.

Desjardins, X. (dir. P. Boino) (2006). L'intercommunalité et décentralisation. Paris : Plan Urbanisme Construction Architecture.

(2009). Dossier presse 40 ans du Grand Lyon 1969-2009, Rendons service à l'avenir.

Gazendam, H. W.M. (2003). Models as coherent sign structure. Gazendam, H. W. M., Jorna R. J. \& Cijsouw, R. S. (Eds.), Dynamics and change in organizations: Studies in organizational semiotics. Dordrecht : Kluwer Academic Publishers.

Jalenques-Vigouroux, B. (2006). Dire l'environnement : le métarécit environnemental en question. thèse de doctorat en Sciences de l'information et de la communication, CELSA, Université Paris IV Sorbonne.

Krieg-Planque, A. (2009). La notion de "formule » en analyse de discours. Dijon : PUFC.

Lefèvre, P. (2008). Voyages dans l'Europe des villes durables. Paris : PUCA CERTU.

Libaert, T. (2008). Communiquer dans un monde incertain. Paris : Ed. Pearson.

Libaert, T. (2010). Communication et environnement, le pacte impossible. Paris : PUF.

Maingueneau, D. (1998). Analyser les textes de communication. Paris : Dunod.

Porcedda, A. (2009). Musées et développement durable, les muséums nature de Montréal. Paris : L'Harmattan.

Tremblay, S. (Ed.) (2007). Développement durable et communications. Au-delà des mots, pour un véritable engagement. Québec : Presses universitaires de Québec. 